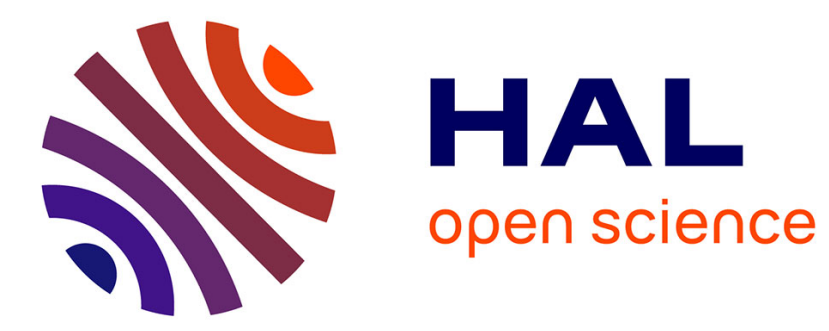

\title{
A digital image correlation method for tracking planar motions of rigid spheres: application to medium velocity impacts
}

\author{
Jean-Charles Passieux, Pablo Navarro, Jean-Noël Périé, Steven Marguet, \\ Jean-François Ferrero
}

\section{To cite this version:}

Jean-Charles Passieux, Pablo Navarro, Jean-Noël Périé, Steven Marguet, Jean-François Ferrero. A digital image correlation method for tracking planar motions of rigid spheres: application to medium velocity impacts. Experimental Mechanics, 2014, 54 (8), pp.1453-1466. 10.1007/s11340-014-9930-y . hal-01025172v2

\author{
HAL Id: hal-01025172 \\ https://hal.science/hal-01025172v2
}

Submitted on 29 Jul 2014

HAL is a multi-disciplinary open access archive for the deposit and dissemination of scientific research documents, whether they are published or not. The documents may come from teaching and research institutions in France or abroad, or from public or private research centers.
L'archive ouverte pluridisciplinaire HAL, est destinée au dépôt et à la diffusion de documents scientifiques de niveau recherche, publiés ou non, émanant des établissements d'enseignement et de recherche français ou étrangers, des laboratoires publics ou privés. 


\title{
A digital image correlation method for tracking planar motions of rigid spheres:
}

\section{application to medium velocity impacts}

\author{
J.-C. Passieux - P. Navarro • J.-N. Périé •
}

S. Marguet • J.-F. Ferrero

Current draft version: July 29, 2014

\begin{abstract}
This paper deals with the measurement of spherical impactor positions, velocities and acceleration (translations and rotations) during medium velocity impact experiments performed with gas-gun devices. A dedicated 2D Digital Image Correlation (DIC) technique based on customized interpolation functions is presented. The proposed method considers the rotations of the projectile which a standard subset-based DIC technique would undoubtedly have difficulty managing. Emphasis is placed on metrological performance and various validations are proposed. Measurements are additionally compared to those retrieved with conventional techniques. This DIC method provides a precise quantification of projectile motion and impact loads during gas-gun tests with a single high speed camera.
\end{abstract}

Keywords optical flow · motion analysis - impact force · medium velocity impact $\cdot$ object tracking

\section{Introduction}

Predicting the structural integrity of composite structures under impact is still a challenge and specific models have been developed for the simulation of damage Université de Toulouse; ICA (Institut Clément Ader), INSA/UPS/Mines Albi/ISAE, 135 avenue de Rangueil F-31077 Toulouse, France

J.-C. Passieux

E-mail: passieux@insa-toulouse.fr 
and delamination $[46,22,16,27,14,2]$. To validate the model, and, potentially, to identify some of its constitutive parameters, a few well instrumented tests must be performed. Thus, accurate instrumentation techniques are indispensable. In the particular case of impact tests, some of the most important information concerns the projectile motion (position, velocity, rotation, acceleration) and the time evolution of the impact loading. These data may be hard to obtain in the medium velocity range.

First, dynamic impact forces can be recovered by using strain gauges on the target $[11,9,48]$. Embedded electrical [38], piezoelectric [20] or fiber optic [45,3] sensors can also be integrated during the manufacturing of the composite specimen for estimating impact load. These methods often rely on the assumption that the impacted structures show elastic behaviour, so that it is possible to use Green's functions, for example [21]. Consequently they are not suitable for impacts on complex structures.

Then, the impact load is generally estimated by measuring the deceleration of the projectile during the impact. An accelerometer is sometimes placed on the impactor, but its use is restricted to low velocity impacts performed with drop weight devices [7] and the projectiles cannot be easily instrumented with an accelerometer in the context of medium velocity impacts obtained with gas-guns.

Another way to measure the deceleration without instrumenting the impactor is to use optical interferometry systems (e.g. VISAR [4]). These techniques allow velocities to be measured with very good spatial and temporal resolutions [47]. However, alhough this technique is suitable for tracking projectiles travelling along a rectilinear path, the use of VISAR for more complex impacts such as impacts on an oblique target does not seem appropriate.

Finally other measurement techniques use high-speed digital cameras [37]. A recorded sequence of images is analysed using image analysis. Tracking one or more objects in an image is a challenging problem for which many techniques have been developed. They can be based on points, primitive geometric shapes or contours and can rely on colour, gradients (edges), or patterns ; see [50] for a survey. For example, the Hough transform [19] is able to locate circles in an image. It may detect the edges of the projectile on the basis of image gradient. Unfortunately, as will be shown in the examples below, the measurement uncertainties are often too 
large to allow acceleration to be estimated by double differentiation [1]. Worse, the deformation of the target sometimes (for instance in oblique impact) results in projectile-to-target occlusions, which even limit the use of such methods. In addition, edge detection algorithms can not retreive rotation velocity which is a mechanical quantity of interest in oblique impacts for instance. Other methods are based on the tracking of markers. However, these methods are difficult to use in the context of impacts with gas-guns since the orientation of the projectile cannot be precisely controlled.

In this paper, the possibility of measuring the impact force accurately by using optical flow [18] referred to as 2D-DIC [26,43] is investigated. To implement this, paint is sprayed onto the surface of the spherical impactor in order to obtain contrasted random speckle in the region of interest (ROI). As the trajectory of the sphere centre is assumed to be planar in the impact tests, only one high-speed digital camera is required (frontal parallel configuration).

In the literature (see [29] for a review), digital image correlation most frequently refers to the so-called subset-based approaches that underlie most commercial DIC packages. The idea is to pair up local zones of interest (ZOI: subsets of the ROI) which usually consist of rectangular windows. The method tries to find the coefficients defining the transformation of the ZOI $[26,44,43]$ between the reference and the deformed images. Optical flow has already been used for object tracking with motion models which may be constant (translations) [26], bilinear [44] or affine [15]. These methods are not optimal for our application since (a) they do not take advantage of the rigid body assumption (which may be used as a regularisation) since measurements are independant from one local ZOI to the other and (b) the impactor may undergo full 3D rotations that are not taken into account by the above motion models if a single ZOI is analysed.

In this paper, a dedicated DIC technique is proposed for tracking the rigid body motion of the impactor within a single zone of interest. This constraint acts as a regularization that makes the method more robust with respect to noise. Even if it is possible to estimate it with a monocular view [35,32], the out-ofplane translation is assumed negligible in this application. Therefore, the method consists of looking for displacement in a region of interest covering the entire visible part of the impactor. The global motion model is entirely described by five 
parameters. The first two correspond to in-plane rigid body translations and the others correspond to the angles of the three rigid body rotations.

The method is compared with conventional measurement techniques: a standard translation-based DIC technique, a circle-center tracking algorithm, a lightgate and an accelerometer, on both real and synthetic experiments. As an example, the method is applied to a classical impact test, with simplified metallic targets. The measured velocity and impact force are compared to numerical simulations using nonlinear explicit transient dynamics and modal analysis.

The paper is organized as follows. The proposed digital image correlation method is explained in Section 2. Sensitivity to noise and grey level interpolation are analysed in Section 3. The sensitivity to small rotations and motion blur are also discussed. In section 4 , the method is compared to conventional measurement techniques on real experiments. The method is exemplified on a classical impact test, with simplified metallic targets in Section 5. Finally, a last oblique impact is analysed, in section 6.

\section{Principle of the proposed DIC technique}

\subsection{Digital image correlation}

The proposed method is based on the $2 \mathrm{D}$ correlation of mono-vision digital images $[44,39,17,33]$. A pair of images $f$ and $g$, taken at times $t$ and $t+\Delta t$ respectively, is considered. During the time interval $\Delta t$, the projectile is assumed to undergo rigid body translations and rotations. The corresponding displacement $\mathbf{u} \in \mathbf{L}^{2}(\Omega)$ results in a variation of grey levels within the region of interest $\Omega$, such that the grey level conservation assumption [18] is satisfied:

$$
f(\mathbf{x})=g(\mathbf{x}+\mathbf{u}(\mathbf{x}))
$$

where $\mathbf{x}$ is the position in the image. To measure a non-integer displacement, an interpolation scheme of the grey level between pixels has to be used. A spline interpolation is employed here [41]. Classically, the grey level conservation is written in a least square sense [44]: find $\mathbf{u}(\mathbf{x}) \in \mathbf{L}^{2}(\Omega)$ minimizing the sum of squared 
differences $\phi^{2}$

$$
\phi^{2}=\int_{\Omega}[f(\mathbf{x})-g(\mathbf{x}+\mathbf{u}(\mathbf{x}))]^{2} d \mathbf{x}
$$

Remark. In practice, since offset and scale changes in illumination light may occur, such a simple criterion is not applied directly $[8,43,30]$. The advected deformed image $g(\mathbf{x}+\mathbf{u}(\mathbf{x}))$ is scaled $a$ and offset $b$ in order to make sure that the mean value and standard deviation of $g(\mathbf{x}+\mathbf{u}(\mathbf{x}))$ and $f(\mathbf{x})$ are equal within the ROI. Therefore, the used criterion reads:

$$
\phi^{2}=\int_{\Omega}[f(\mathbf{x})-a \cdot g(\mathbf{x}+\mathbf{u}(\mathbf{x}))-b]^{2} d \mathbf{x}
$$

In the following, $a$ and $b$ will be omitted for the sake of clarity.

This nonlinear problem is solved using an iterative process. At iteration $k$, an approximation of the displacement at the previous iteration $\mathbf{u}_{k-1}(\mathbf{x})$ is assumed to be known. The problem is to correct this approximation $\mathbf{u}_{k}(\mathbf{x})=\mathbf{u}_{k-1}+\delta_{k} \mathbf{u}(\mathbf{x})$. The correction $\delta_{k} \mathbf{u}(\mathbf{x}) \in \mathbf{L}^{2}(\Omega)$ (which is refered to as $\delta \mathbf{u}$ in the following for more clarity) is assumed to be small enough to allow the use of a first order Taylor expansion:

$$
g(\mathbf{x}+\mathbf{u}(\mathbf{x})) \approx g\left(\mathbf{x}+\mathbf{u}_{k-1}(\mathbf{x})\right)+\delta \mathbf{u}^{T} \nabla g\left(\mathbf{x}+\mathbf{u}_{k-1}(\mathbf{x})\right)
$$

Since gray level conservation equation (1) should be verified (in a least-square sense (2)) once convergence has been reached, the gradient of image $g$ at the position $\mathbf{x}+\mathbf{u}(\mathbf{x})$ should be equal to the gradient of image $f$ at point $\mathbf{x}$. Thus $\nabla g(\mathbf{x}+\mathbf{u}(\mathbf{x}))$ is classically approximated by $\nabla f(\mathbf{x})[5,39,17]$. This approximation has effect on the convergence rate only, and allow to compute once and for all the gradient of $f$, and therefore the correlation operator. Introducing this approximation in problem (2) yields the linearized problem:

$$
\phi^{2}=\int_{\Omega}\left[\left(f-g_{u}\right)-\delta \mathbf{u}^{T} \boldsymbol{\nabla} f\right]^{2} d \mathbf{x}
$$

where $g_{u}(\mathbf{x})=g\left(\mathbf{x}+\mathbf{u}_{k-1}(\mathbf{x})\right)$ and $\left(f-g_{u}\right)$ is the discrepancy map.

The stationary conditions corresponding to the minimization of (5) yield the variational formulation of the digital image correlation problem[12]:

$$
\text { find } \delta \mathbf{u} \in \mathbf{L}^{2}(\Omega) \quad a(\delta \mathbf{u}, \delta \mathbf{v})=L(\delta \mathbf{v}) \quad \forall \delta \mathbf{v} \in \mathbf{L}^{2}(\Omega)
$$


where $\delta \mathbf{v} \in \mathbf{L}^{2}(\Omega)$ is a test function and

$a(\delta \mathbf{u}, \delta \mathbf{v})=\int_{\Omega}\left(\delta \mathbf{u}^{T} \boldsymbol{\nabla} f\right)\left(\delta \mathbf{v}^{T} \boldsymbol{\nabla} f\right) d \mathbf{x} \quad$ and $\quad L(\delta \mathbf{v})=\int_{\Omega}\left(\delta \mathbf{v}^{T} \boldsymbol{\nabla} f\right)\left(f-g_{u}\right) d \mathbf{x}$

The dimension of space $\mathbf{L}^{2}(\Omega)$ being infinite, an approximation $\delta \mathbf{u}^{h}$ of $\delta \mathbf{u}$ is sought in an approximation subspace $\mathcal{U}^{h} \subset \mathbf{L}^{2}(\Omega)$ and is defined by:

$$
\delta \mathbf{u}^{h}(\mathbf{x})=\sum_{n=1}^{N} \boldsymbol{\varphi}_{n}(\mathbf{x}) q_{n}
$$

where $\varphi_{n}$ are given interpolation basis functions, $q_{n}$ is the corresponding degree of freedom (dof), and $N$ is the dimension of $\mathcal{U}^{h}$.

The use of such an interpolation (8) in Problem (6) leads to the resolution of the following linear system at iteration $k$ :

$$
\mathbf{M} \mathbf{q}^{k}=\mathbf{b}^{k}
$$

where $\mathbf{q}$ is the dof vector collecting the values $q_{n}$. Correlation operator $\mathbf{M}$ and right-hand side vector $\mathbf{b}^{k}$ are defined by:

$$
\mathbf{M}_{i j}=a\left(\boldsymbol{\varphi}_{i}(\mathbf{x}), \boldsymbol{\varphi}_{j}(\mathbf{x})\right) \quad \text { and } \quad \mathbf{b}_{i}^{k}=L\left(\boldsymbol{\varphi}_{i}(\mathbf{x})\right)
$$

Remark: The image must be textured so that system (9) is not singular [12]. If the texture is almost uniform, its gradient $\nabla f$ vanishes, and the system becomes singular. So the impactor is usually covered with black and white paint, in order to apply random speckle to its boundary, following the recommendations of [43].

\subsection{The proposed rigid body interpolation}

At this stage, neither the choice of the interpolation functions $\varphi_{n}(\mathbf{x})$ nor the region $\Omega$ have been specified. In the literature, the interpolation functions most often have a locally bounded support (Zone Of Interest or subset) and they consist of a set of piecewise constant or piecewise polynomial functions [26,44]. More recently, global DIC methods have been proposed. When a continuous displacement field is expected, global continuous basis functions, for instance Fourier series [40] or B-Splines [10], could be used. Another choice for the displacement interpolation can be to resort to the finite element framework $[42,5,13]$. To keep the advantages 
of the former method and reduce the associated computational time, a formulation based on the Proper Generalized Decomposition (PGD) has also been proposed recently [33,34]. Lastly, when a priori relevant mechanical information is available, analytical [39] or numerical precomputed basis functions can also be used [24]. In the following, the technique is applied in the case of a spherical impactor. As mentioned above, the projectile is assumed to undergo rigid body translations and rotations between two images. Even though it would be possible to measure it with a single camera [35,32], the translation along the $z$-axis (out-of-image-plane vector) is assumed to be negligible in this study. Therefore, the motion model is based on five parameters - the two in-plane translations and the three rotation angles_- The measurement is performed within a single circular region of interest $\Omega$ corresponding to the orthogonal projection of the ball onto the image plane. Only 5 parameters need to be optimized to characterize the whole motion of the impactor between two consecutive images. In the following, this method will be compared to a more standard one based on the two in-plane translations only. Such a technique can be carried out with standard DIC packages, provided that they could use a circular ZOI.

More precisely, as the displacement is assumed to be small between two images, the orthogonal projections of linearized rotations are used as basis functions. Large rotation could also be considered [32] but, in this application, the rotation angle between two consecutive images is smaller than one degree. Five basis functions are defined within a circular region of interest (ROI) of radius $R$ centred on the ball centre $C\left(x_{c}, y_{c}\right)$ as follows: $\forall$ pixel $(x, y)$ such that $\left(x-x_{c}\right)^{2}+\left(y-y_{c}\right)^{2}<R^{2}$,

$$
\begin{aligned}
& \varphi_{1}(x, y)=\left(\begin{array}{l}
1 \\
0
\end{array}\right) \quad \varphi_{2}(x, y)=\left(\begin{array}{l}
0 \\
1
\end{array}\right) \\
& \varphi_{3}(x, y)=\left(\begin{array}{c}
\sqrt{1-\frac{\left(x-x_{c}\right)^{2}}{R^{2}-\left(y-y_{c}\right)^{2}}} \\
0
\end{array}\right) \quad \varphi_{4}(x, y)=\left(\begin{array}{c}
0 \\
\sqrt{1-\frac{\left(y-y_{c}\right)^{2}}{R^{2}-\left(x-x_{c}\right)^{2}}}
\end{array}\right) \\
& \boldsymbol{\varphi}_{5}(x, y)=\left(\begin{array}{c}
y_{c}-y \\
x-x_{c}
\end{array}\right)
\end{aligned}
$$

A graphical representation of the vector fields associated to the five modes is given in Figure 1 


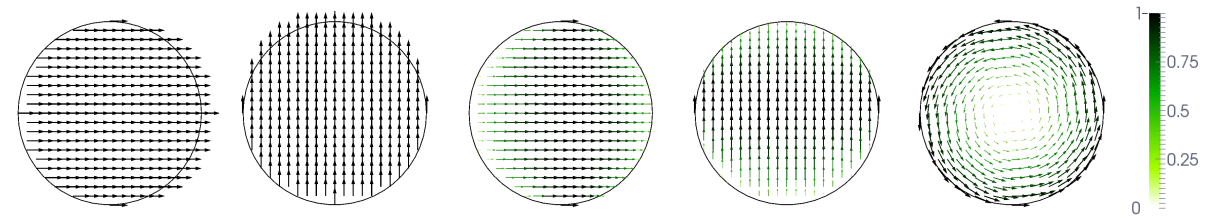

Fig. 1 Five rigid body translations and rotations used as a displacement basis for the spherical projectile with a radius corresponding to 10 pixels. From left to right: $x$-axis translation, $y$-axis translation, $y$-axis rotation, $x$-axis rotation and $z$-axis rotation. The colormap corresponds to the amplitude of the vector field.

Remark. A simple orthogonal projection was used even though the image acquisitions were carried out with a standard lens (telecentric lenses could also be used for more accuracy [43,31]). A perspective projection based on a pinhole camera model has also been considered $[49,43]$ but, according to our tests, it does not significantly improve the measurement accuracy. Distortion could also be taken into account, but the authors did not observe significant evolution of the velocity as a function of the position in the image during free-fall tests.

The high speed digital camera provides a sequence of $N_{i}$ images $\left\{f_{i}\right\}_{0 \leq i \leq N_{i}}$. The procedure described in section 2.1 is used to measure the displacement of the ball between two consecutive images, namely with $f=f_{i}$ and $g=f_{i+1}$. Thus, the measured quantity is a set of displacement increments, which, when divided by the time lapse between two images, correspond to the velocity.

Finally, since the sequences analysed have many images, the initial ROI provided by the user is updated automatically using the displacement measured previously.

\subsection{Implementation}

The proposed digital image correlation method was implemented in a global DIC research code (developed in a previous study [33]) using the cross-platform programming language Matlab (http://www.mathworks.com). The .tif (Tagged Image File Format) images were imported from the digital camera by the native image reading/writing function of Matlab. The symmetric system (9) was solved by a direct LU solver. The results were displayed using both Matlab and Paraview, an open-source, cross-platform data analysis and visualization application 
(http://www.paraview.org/). As regards run time, it took 43s to analyse a sequence of 114 images which corresponds to $0.38 \mathrm{~s}$ to compute the unknown displacement between two images. This run time obviously depends on the image resolution $(256 \times 512$ here) and hardware (standard laptop, in our case).

\section{Validation with synthetic images}

In this section, the capacities of the method are evaluated in order to optimize the experimental parameters such as shutter speed, frame rate, image resolution, optical resolution (i.e., number of pixels in the ROI) and lighting to name but a few. Particular attention is paid to the sensitivity to noise and grey level interpolation. The ultimate and asymptotic error regimes described in [5,6] are studied in sections 3.1 and 3.2 respectively. The effect of motion blur due to the velocity of the projectile is also investigated. For that purpose, synthetic images are generated from a real reference image by spline interpolation and inverse mapping as described in $[28,36]$.

3.1 Effect of the rotation of the impactor

As mentioned before, the ball may undergo some rotations during the time lapse between two consecutive images. The aim of this section is to quantify the systematic error (i.e. bias) as a function of the rotation amplitude. A DIC method considering only in-plane translations and the proposed DIC technique also including rotations are compared. To come close to the testing conditions, a still image of the spherical impactor taken from a movie of a real experiment is used as a reference image. This image $f$ is then advected numerically for a displacement field involving an $x$-axis rigid body shift and a linearized $y$-axis rotation (with angles ranging from 0 to 1 degree). For each value of the rotation angle, the value of the bias on the $x$ component of the displacement (averaged over 20 values of the shift ranging from 0 to 1 ) is given in Figure 2(left). It can be observed that, (a) when there is no rotation in the actual displacement field, a translation-based interpolation gives a much lower bias on the displacement; and (b) when the ball is subjected to any non-zero rotation, the bias on the displacement becomes more 

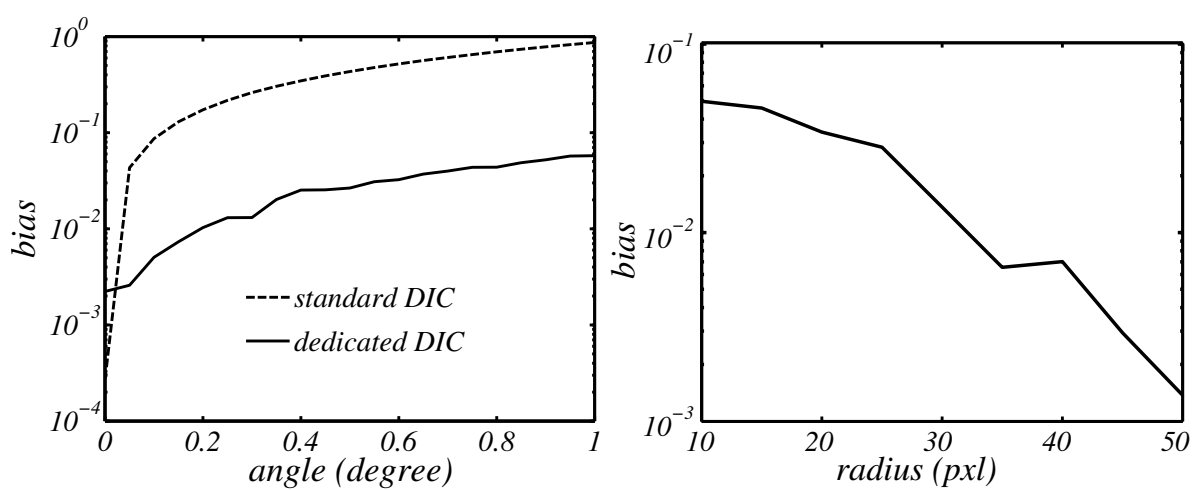

Fig. 2 Bias on the displacement according to the angle (left) and its variation with the size of the ball for a standard DIC and with the proposed interpolation.

than two orders of magnitude bigger. The can be explained by the fact that, when looking Figure 1, a $y$-axis rotation differs from a $x$-axis translation at the left and right edges of the disk only. (the same kind of observation apply with $y$-translation and $x$-rotation).

(a) when there is no rotation, the translation-based DIC has lower uncertainties than the one including rotations. This is due to the fact that there are two pairs of modes that are very similar. This yields a worse condition number for $M$ when using rotations. The problem being ill posed, a worse condition number implies higher measurement uncertainties.

(b) when the ball is subjected to a rotation of any non-zero angle, the translationbased displacement interpolation is not consistent with the actual motion. The algorithm tries to best match two consecutive images with only rigid body translations. Since the translation along $x$ is almost identical to the rotation along $y$ (except on the edges), the translation-based DIC interpret the rotation by a rigid body translation. This results in a large over or under estimation of the translation. Since the proposed method is able to make the distinction between $x$-translation and $y$-rotation (for instance), the measurement is more accurate.

The standard translation-based interpolation should not be used for tracking an impactor if the latter undergoes even very small rotations since the kinematic model error becomes prohibitive [6]. 
3.2 Choice of the image resolution

The choice of the image resolution is the result of a compromise between spatial and temporal resolutions, namely the image resolution and the frame rate [37]. This compromise is necessary because of the limitation on data throughput of the digital high-speed cameras used. In practice, image resolution must be lowered to improve temporal resolution (higher frame rate).

In the present section, the effect of the size of the spherical impactor (expressed in pixels) on the measurement accuracy is analysed for both interpolations in the asymptotic regime. The asymptotic regime is reached when the chosen interpolation is consistent with the actual displacement field. Thus synthetic images are generated with rigid body translations. A set of synthetic distorted images $g_{k}$ are generated by numerical advections of the reference image $f$ (the same image as previously): 20 rigid body translations from 0 to 1 pixel are applied in the $x$ direction. Measurements are carried out with 9 circular masks covering from 10 to 50 pixels. The bias between the prescribed and the measured translation (averaged over the 20 shift values) is plotted versus the ball radius (expressed in pixel) in Figure 2(right) for the proposed interpolation. As expected [43], the bias decreases when the number of pixels in the ROI increases.

3.3 Shutter speed and sensitivity to motion blur

Shutter speed is the result of a compromise between contrast (or lighting) and motion blur. The effect of motion blur on the accuracy of the measurement is studied here. An image of a static ball (with no motion blur) is used to synthesize various reference images including distinct amplitudes of motion blur following [25]. The effect of motion blur is then analysed with respect to noise and sub-pixel interpolation sensitivity.

Sensitivity to noise. Random noise with a standard deviation $\sigma$ ranging from 1 to 8 grey levels is added to the reference image ( 8 bits). For each image generated, motion blur 2 to 20 pixels wide is added. The images are not advected by any displacement field, so the measured rigid body translation should be identically equal to zero. Figure 3(left) presents the value of the bias averaged over the different 
values of $\sigma$ as a function of the blur width. The sensitivity to noise increases as the blur becomes wider.
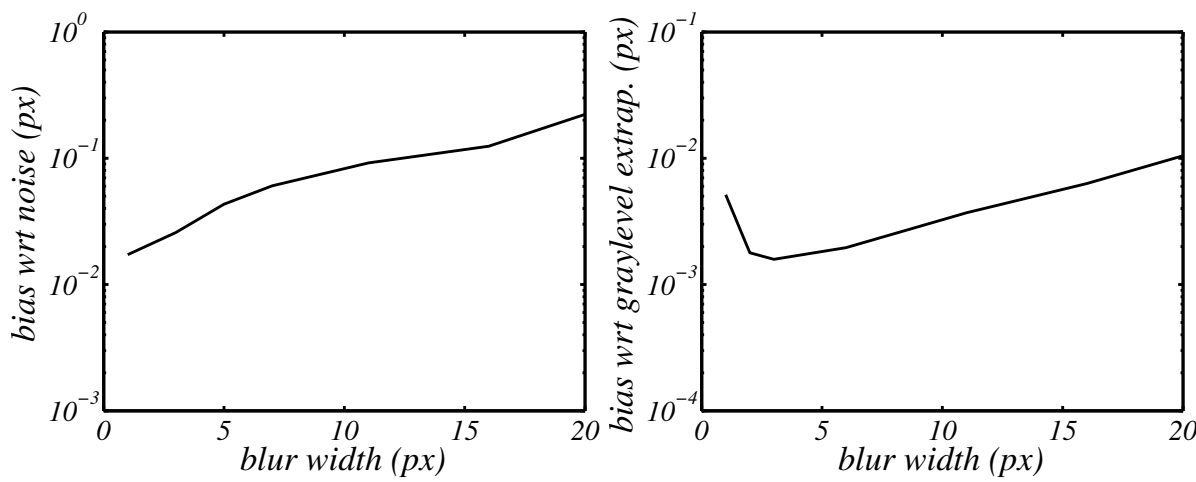

Fig. 3 Effect of blur width on the sensitivity to noise (left) and grey level interpolation (right) with the proposed DIC interpolation

Sensitivity to grey level interpolation. The reference image is advected by a rigid body translation in the $x$ direction from 0 to 1 pixel. Again, for each image generated, motion blur 2 to 20 pixels wide is added. Figure 3(right) presents the value of the bias averaged over the different shift values versus the blur width. The sensitivity to grey level interpolation begins to decrease for small values of the blur, and then increases when the blur becomes wider. This result could be expected since the motion blur smoothes the grey level distribution. Consequently, even a linear interpolation would be accurate. When the blur becomes too wide, the gradient of the image becomes smaller and the linear system becomes illconditioned.

In practice, the shutter speed was set so as to obtain a motion blur below 5 pixels. The motion blur width $B_{w}$ was estimated by $B_{w}=v_{b}^{i} \cdot t_{e} \cdot p_{m}$, where $v_{b}^{i}$ is the initial velocity of the ball, $t_{e}$ is the inverse of the shutter speed and $p_{m}$ is the size of one pixel in metres.

The conclusion is that such an a priori analysis should be performed before any real impact test. It requires only one experiment with no target. The experimenter can thus make sure that the measurement uncertainties are compatible with the expected magnitude of the measured quantities. If this is not the case, he will have to adapt the image resolution, frame rate, shutter speed or lighting accordingly. 
4 Validation with real experiments and comparison with conventional measurements

Here, in contrast to the previous section, the method is validated on real experiments. The method is compared with three other conventional measurement techniques: a light-gate to measure the initial velocity, an accelerometer to measure the variation of the acceleration of the impactor, and a contour tracking technique like the Hough transform to estimate both velocity and acceleration.

4.1 Velocity: comparison with light-gate

For this comparison, a real experiment with a gas-gun device was performed. A steel ball covered with black and white speckles was fired. The trajectory (assumed rectilinear) of the projectile was filmed with a Photron APX-RS CMOS high speed digital camera (36000 fps). The image sequence was analysed a posterior $i$ with the proposed digital image correlation method. In this section, the estimated velocity is compared to a measurement made using light-gate, a system composed of two light barriers (photodiode and phototransistor) spaced $d x=40 \mathrm{~mm}$ apart. They can detect objects that pass between the emitter and the receiver through the interruption of the light beam. The tension across the receiver is recorded during the test, see Figure 4(left). A 1D DIC-like algorithm was used to precisely measure the time lapse $d t$ between the signals recorded by the two light barriers. The velocity was finally estimated by $v=d x / d t$. The average velocities measured by DIC are reported in Figure 4(right) for two values of pressure in the gas-gun. The error bars, corresponding to the standard deviation of the velocity variation, provide an idea of the measurement uncertainty.

The velocities measured by the proposed DIC method are in good agreement with the light-gate measurement as shown in Table 1 where the difference between the velocity estimated by DIC and the light-gate measurement is less than the estimated measurement uncertainty, and also certainly less than the light-gate measurement uncertainty. This is the only conclusion that can be drawn but it is, nevertheless, a convincing result. 

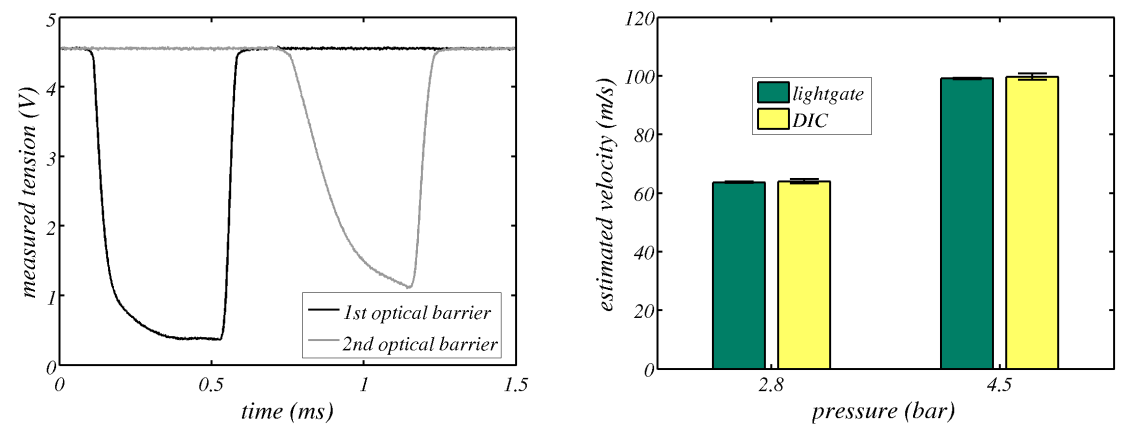

Fig. 4 Variation of the voltage measured across the two receivers (left) and velocity estimated by the DIC method as compared to light-gate measurement for two different velocity ranges (right).

Table 1 Mean values and standard deviations of the velocity estimated by DIC and relative gap with respect to the velocity estimated by light-gate for two different pressures of the gas-gun.

\begin{tabular}{|l|l|cc|}
\hline $\begin{array}{l}\text { Pressure } \\
(\mathrm{bar})\end{array}$ & light-gate & \multicolumn{2}{|c|}{ DIC (m/s) } \\
$(\mathrm{m} / \mathrm{s})$ & mean $\pm \mathrm{std}$ & discrepancy \\
\hline \hline 2.8 & 63.63 & $63.99 \pm 0.79$ & $0.57 \%$ \\
4.5 & 99.12 & $99.70 \pm 1.05$ & $0.61 \%$ \\
\hline
\end{tabular}

\subsection{Acceleration: comparison with an accelerometer}

As stated in the introduction, it is easier to embed an accelerometer in the impactor of a drop weight device than in a gas-gun projectile. Indeed, for medium velocity impacts produced by a gas-gun it would be prohibitively expensive to build a 19$\mathrm{mm}$ ball with a battery-powered high-g accelerometer and data acquisition system inside. Thus a low velocity impact was obtained with a drop weight on a wooden plate in order to compare the acceleration measured by the proposed method with that given by an embedded accelerometer. The hemispherical head of the impactor was covered with a black and white pattern, and the scene was filmed with the same digital camera (5000 fps). The variation of the acceleration is shown in Figure 5. The acceleration measured by DIC is in good agreement with the accelerometer measurement which is, however, quite noisy. Both the amplitude and duration of the impact lobe are well measured. The results given by the two methods are in concord, which confirms that the proposed method is robust and reliable. 

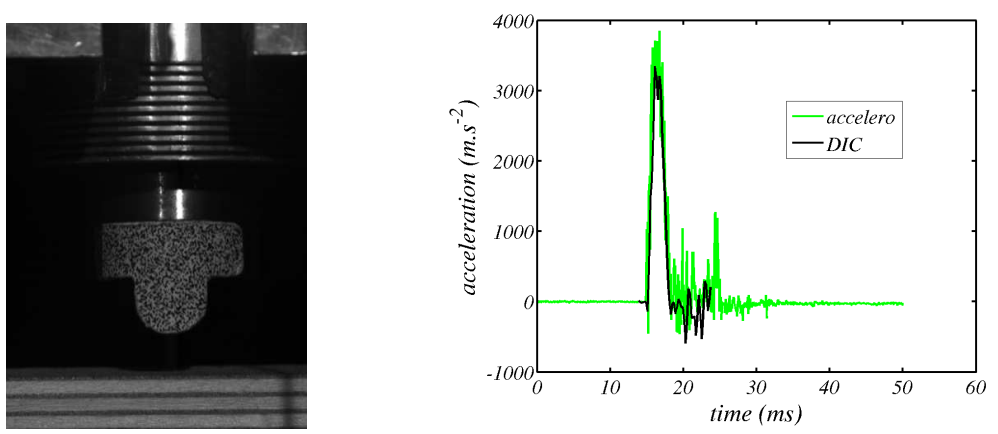

Fig. 5 (left) drop weight test setup and (right) comparison between the acceleration estimated with the proposed DIC technique and that recorded by the accelerometer.

4.3 Velocity and acceleration: comparison with a contour tracking algorithm

In this section, the proposed DIC based method is compared to an implementation of the circular Hough transform [19]. The latter was analysed using the imfindcircles function of Matlab's Image processing toolbox. For that purpose, a real experiment was carried out. A spherical impactor was fired from the gas-gun device without a target, so the velocity was assumed constant $\left(v_{X} \approx 100 \mathrm{~m} / \mathrm{s}\right.$ and $v_{Y} \approx 0 \mathrm{~m} / \mathrm{s}$ ). A first ball was covered with a uniform coast of white paint and the images were analysed with the circular Hough transform. Then the experiment was performed again with black and white random speckle covering the impactor, the corresponding images being analysed by the proposed DIC technique, see Figure $6($ left).

The variation of the verical and horizontal components of the velocity measured by both techniques are reported in Figure 6(right). This qualitative result shows that the estimation of the velocity seems more scattered with the circular hough transform.

Remark: Note that authors did not manage to make the circular hough transform locate the sphere center when the background was not uniform, this is why there is no data before $0.75 \mathrm{~ms}$ with the method based on the Hough transform.

Table 2 shows that the standard uncertainty on velocity is 3 to 4 times smaller with the proposed DIC technique than with a contour tracking technique based on the Hough transform. The standard uncertainty on acceleration is divided by more than 6 when the DIC technique is used. This result is may be explained by 


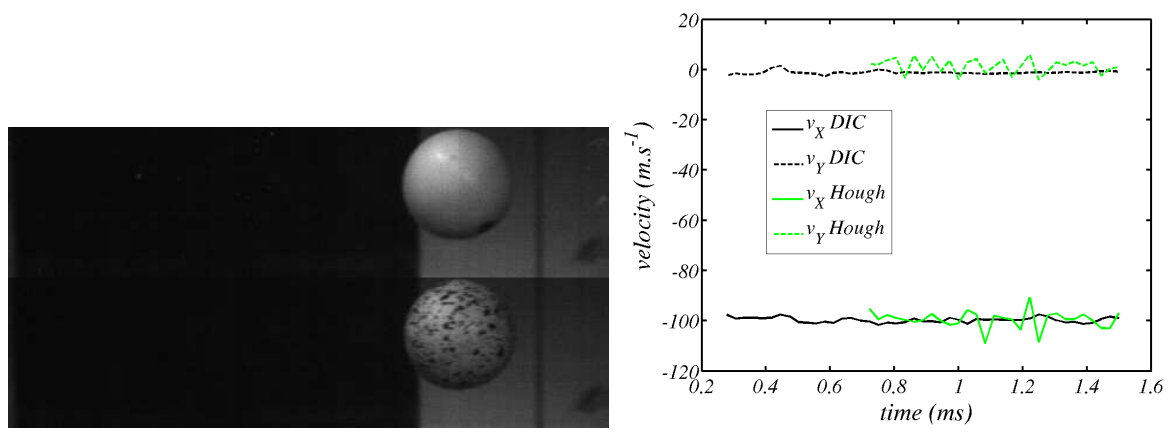

Fig. 6 Images of the impactor with and without speckle pattern (left) and variation of the two components of the velocity measured by both the hough transform based technique (green) and the proposed DIC-based technique (black) (right).

Table 2 Quantification of standard uncertainty on velocity and acceleration: comparison of the proposed DIC technique and a contour detection algorithm based on the Hough transform.

\begin{tabular}{|l|cc|cc|}
\hline & \multicolumn{2}{|c|}{ Velocity $(\mathrm{m} / \mathrm{s})$} & \multicolumn{2}{c|}{ Acceleration $\left(\mathrm{m} / \mathrm{s}^{2}\right)$} \\
& $\operatorname{std}\left(v_{X}\right)$ & $\operatorname{std}\left(v_{Y}\right)$ & $\operatorname{std}\left(a_{X}\right)$ & $\operatorname{std}\left(a_{Y}\right)$ \\
\hline \hline Hough transform & 3.62 & 2.9 & 6.11 & 5.02 \\
Proposed DIC & 1.05 & 0.68 & 0.99 & 0.68 \\
\hline
\end{tabular}

the fact that the Hough transform is based on gradients that only exist at the boundary of the circle, whereas, with the DIC algorithm, all the pixels within the disk contributed to the measurement. Thanks to that property, the proposed DIC method was more accurate than the technique based on the circular Hough transform.

\section{Analysis of a frontal impact test and comparison with simulation}

\subsection{Experimental setup}

An impact test commonly used in the characterization of impacts on composite blades $[27,1]$, was carried out with a horizontally mounted gas-gun. The impact was a frontal impact on a cylindrical bar made of aluminium alloy $(300 \mathrm{~mm}$ long and $18 \mathrm{~mm}$ in diameter). The specimen was positioned perpendicular to the firing axis (see Figure 7 (right)). For this test, the steel ball $(30 \mathrm{~mm}$ diameter and $110 \mathrm{~g}$ weight) was propelled at an average initial velocity of $70 \mathrm{~m} / \mathrm{s}(\approx 270 \mathrm{~J})$. 


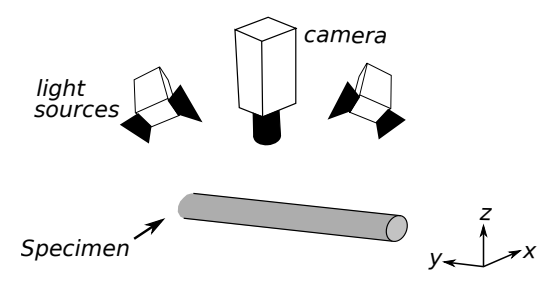

Fig. 7 Setup of the frontal impact test on an aluminium cylindrical bar. Camera is on the top. The ball hits the middle of the bar.

The impactor was a hardened steel ball entirely covered by speckled paint. Images were obtained with the above mentioned camera with the following rate/resolution compromise: $36000 f p s(512 \times 128 \mathrm{px}$ resolution). Three Dedolight (400W) spots are used. The shutter speed was set to $1 / 81000$ s and the estimated motion blur was thus less than 3.2 pixels, while keeping an acceptable contrast.

\subsection{Experimental impact force uncertainty quantification}

In this section, once the acquisition parameters have been chosen, a technique is proposed to estimate a priori standard uncertainties on the velocity and impact force experimentally. The idea is to fire the gas-gun with no specimen in place. Since air friction may reasonably be neglected for such a short travel distance, and since there is no target, the motion is known. In that case, the ball, with a large initial velocity along the $\mathrm{x}$-direction, is considered to be in free fall throughout the image sequence. In practice, the acceleration can be considered negligible and the velocity constant. If there are enough images in the sequence, the mean velocity (and so the acceleration) can be computed with its standard deviation, which provides an estimation of the measurement uncertainty. Thus, it is possible to assess the bias and standard uncertainty by taking all the sources of uncertainties (noise, sub-pixel interpolation, blur etc... ) into account.

Remark. A first impactor was fired without a target for uncertainty quantification, and then a second experiment was carried out with a specimen, using the same impactor. The problem is that, with the gas-gun used, the initial angular position of the impactor could not be controlled experimentally. So even though the same impactor was used, the camera did not see exactly the same side of the im- 
pactor and thus the uncertainty was not quantified in exactly the same conditions as in the real experiment. This problem could be avoided by doing one experiment with two cameras: one filming the impact and the other the pre-impact motion.

Since the ball was not slowed by a target, in these sequences, there was only a limited number (50) of pictures to analyse in the experiment described above. The mean value and the standard deviation of the variation of both components of the velocity and impact force are reported in Table 3. Two features are noteworthy

Table 3 Mean value and standard uncertainty on both components of the velocity and the impact force. The standard uncertainty of the velocity and reaction force in the $x$-direction are also given as a percentage of the measured values $(72 \mathrm{~m} / \mathrm{s}$ and $60 \mathrm{kN})$.

\begin{tabular}{|cc|cc|cc|cr|}
\hline \multicolumn{2}{|c|}{$v_{X}(\mathrm{~m} / \mathrm{s})$} & \multicolumn{2}{c|}{$v_{Y}(\mathrm{~m} / \mathrm{s})$} & \multicolumn{2}{c|}{$F_{X}(\mathrm{kN})$} & \multicolumn{2}{c|}{$F_{Y}(\mathrm{kN})$} \\
mean & std & mean & std & mean & std & mean & std \\
\hline \hline 72.32 & $0.36(<1 \%)$ & 0.72 & 0.27 & 0.0728 & $0.900(1.5 \%)$ & 0.018 & 0.643 \\
\hline
\end{tabular}

in the table. First, the uncertainty in the $x$ direction is always larger than in the $y$ direction. This can be explained by the effect of motion blur which is present in the $x$ direction only. Second, the estimated uncertainty on the impact force is less than $1 \mathrm{kN}$. This value is acceptable with respect to the actual estimated impact force intensity (presented in next section). The standard uncertainties are less than $1.5 \%$ of the measured values.

\subsection{Results and discussion}

The measured positions of the ball are superimposed on the corresponding digital images in Figure 8 for 12 evenly spaced frames taken before, during and after impact. The positions estimated by the proposed DIC technique are plotted with dashed lines. In comparison, the positions estimated by a standard translationbased DIC method are plotted with solid lines. As mentioned in the introduction, because of the contact, the ball undergoes a slight rotation that the standard DIC algorithm does not capture and, in Figure 8, the displacement (and thus the velocity) is underestimated when a basic displacement interpolation is used. On the other hand, it seems that the position is much more accurate with the 


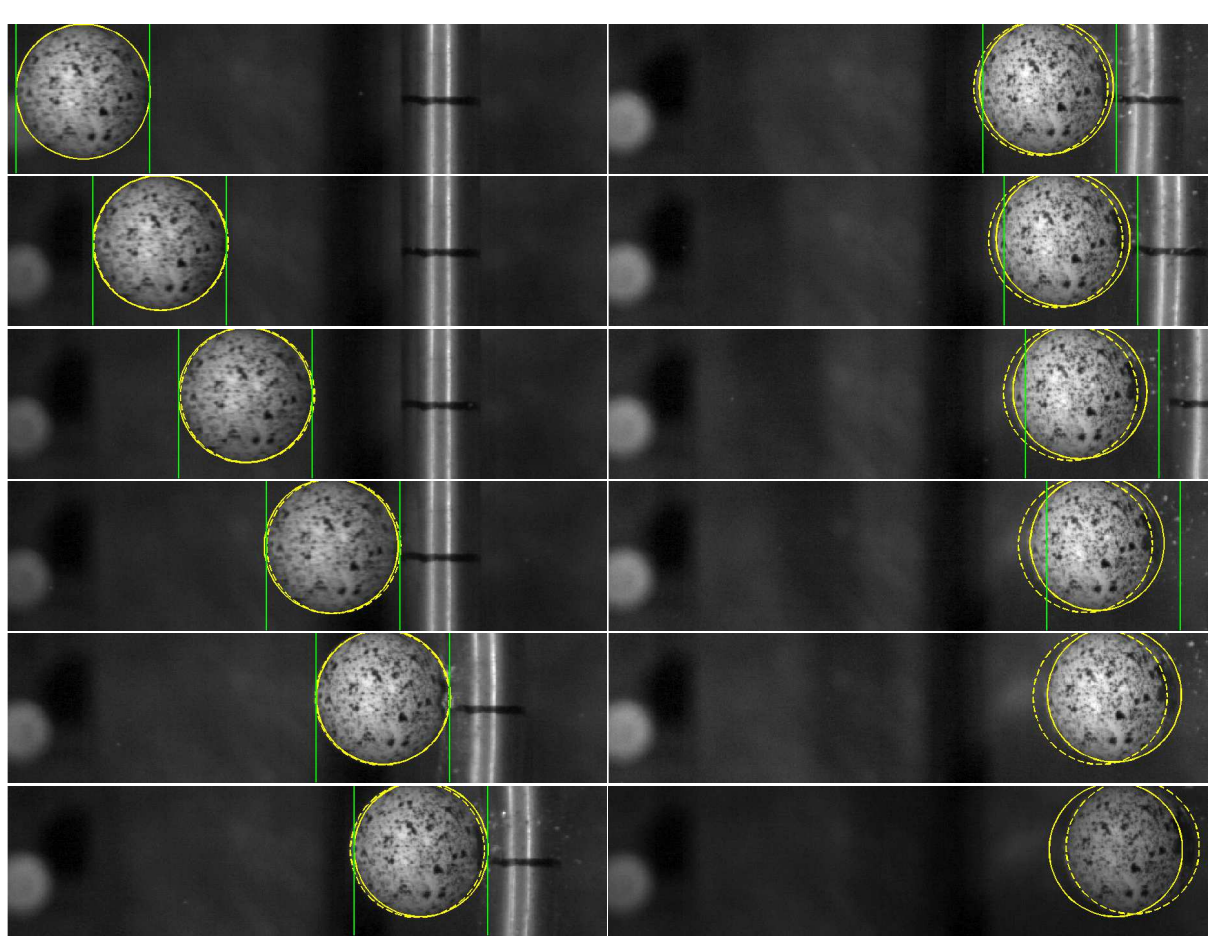

Fig. 8 Positions of the ball measured by the proposed DIC technique (dashed line) as compared to a more standard translation-based DIC technique (solid line) and to a numerical simulation (green lines) for twelve evenly spaced frames, during $3.1 \mathrm{~ms}$

proposed interpolation basis. This may result in a better estimation of the postimpact kinetic energy for instance. Furthermore, the update of the position of the ROI is more accurate. In practice, it was sometimes observed that the standard DIC algorithm lost the impactor due to error accumulation. This was not the case with the proposed algorithm.

The rotation angle and the rotational velocity are also quantities of interest that can be assessed only with the enhanced DIC method. The variation of the three angles and the three angular velocities are plotted in Figure 9. The vertical stripes correspond to an estimation of the time period during which the steel ball is in contact with the target. The value of the time period was estimated by the period of non-zero acceleration. At first, before any contact, the $x$ and $z$ initial rotational velocities are zero whereas the $y$ velocity is not. This corroborates the fact that, even without a target, an instrumentation which takes rotations into 

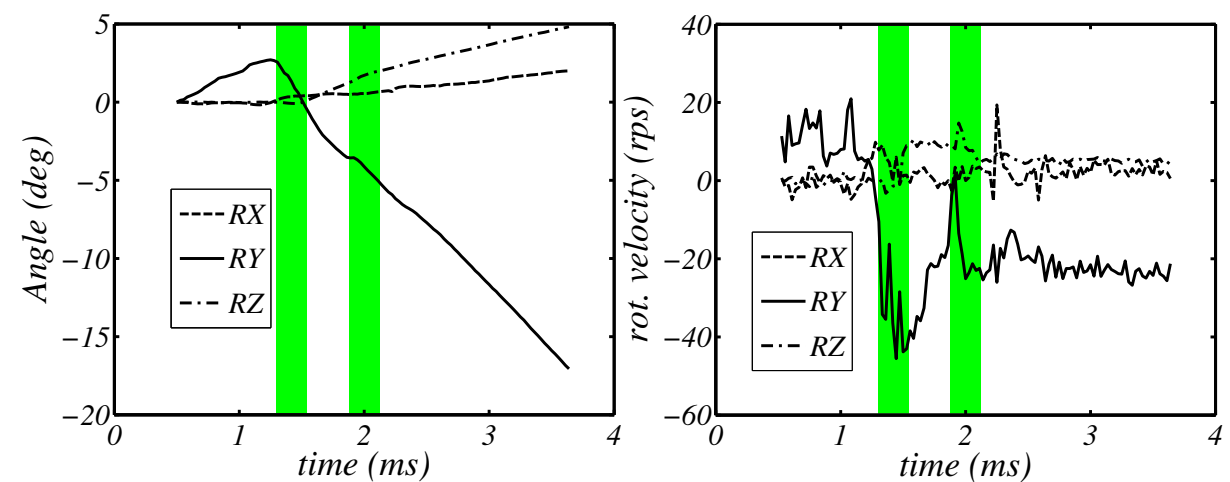

Fig. 9 Variation of the three angular positions (left) and angular velocities (right) during the frontal impact. The vertical stripes correspond to period of contact between the impactor and the target.

account is necessary for any impact test with the present gas-gun device. Next, during impact, the angular velocity undergoes large changes. Thus, not taking them into account seems not accurate, particularly if we are interested in the estimation of the impact force (which, by definition, occurs during the contact period). After the first contact, $x$ and $z$ velocities appear and the rotation angles increase in these directions. The method provides an estimation of the post-impact rotational velocity which seems to be around 20 rotations per seconds (rps). This explains why a standard DIC technique would underestimate the translational velocity and not match the position of the impactor in Figure 8.

\subsubsection{Comparison with numerical modelling}

Here, the frontal impact test is analysed. The results are compared to numerical calculations. Finite element explicit simulations were carried out with the commercial software ABAQUs EXPLICIT.

The impactor was modeled using linear elastic behaviour. The Johnson-Cook model was chosen for the material behaviour of the aluminium bar. When the stress $\sigma$ in the material reaches the yield stress $a$, the constitutive law becomes:

$$
\sigma=\left(a+b \cdot \varepsilon_{p}^{n}\right)\left(1+c \cdot \ln \frac{\dot{\varepsilon}}{\dot{\varepsilon_{0}}}\right)\left(1-\left(\frac{T-T_{i}}{T_{m e l t}-T_{i}}\right)^{m}\right)
$$

where $b$ is the hardening stiffness, $\varepsilon_{p}$ the plastic strain, $n$ a hardening parameter, $c$ the strain rate coefficient, $\dot{\varepsilon_{0}}$ the reference strain rate, $T$ the temperature, $T_{i}$ the 
initial temperature, $T_{m e l t}$ the melting temperature and $m$ a temperature parameter. The material characteristics are given in Table 4. For the impact simulation, the bar was modeled using standard 8-node three-dimensional elements with full integration. The mesh size was smaller in the impacted region (Figure 10). The edge length varied from $0.5 \mathrm{~mm}$ to $15 \mathrm{~mm}$.

Table 4 Material parameters of the Johnson-Cook model chosen to model the behaviour of the aluminium bar.

\begin{tabular}{|c|c|c|c|c|c|c|c|c|c|}
\hline$E(G P a)$ & $\nu$ & $\dot{\varepsilon_{0}}$ & $a(M P a)$ & $b(M P a)$ & $c$ & $n$ & $m$ & $T_{i}(K)$ & $T_{\text {melt }}(K)$ \\
72 & 0.27 & 0.18 & 175 & 223 & 0.1 & 0.37 & 1.34 & 293 & 933 \\
\hline
\end{tabular}
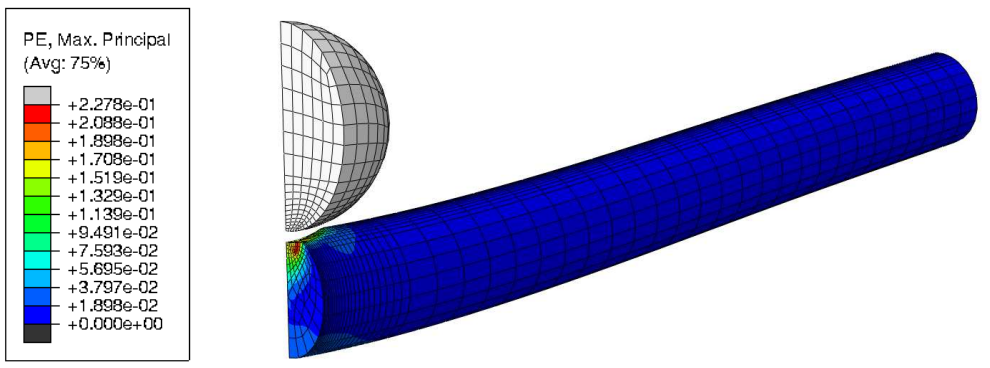

Fig. 10 Modeling of the frontal impact test. The colour map in the target corresponds to maximal principal plastic strain.

The measured impact force and velocity of the impactor during the frontal impact on the aluminium bar are reported in Figure 11 and compared with the numerical simulation (green line). The shapes of the simulated and measured velocities are similar. The mismatch between the measured and simulated post-impact velocity can not be blamed solely on DIC measurement uncertainties. Indeed, when looking at Figure 8, it seems that if computed and measured displacements are in good agreement and are correctly placed in the images before and during impact, the lines corresponding to the simulation clearly diverges from the sphere after impact, while the measurement still locates correctly the ball. The mismatch could also be attributed to some inaccuracies in the FE simulation. According to the impact force, the match between the simulation and the measurement is 

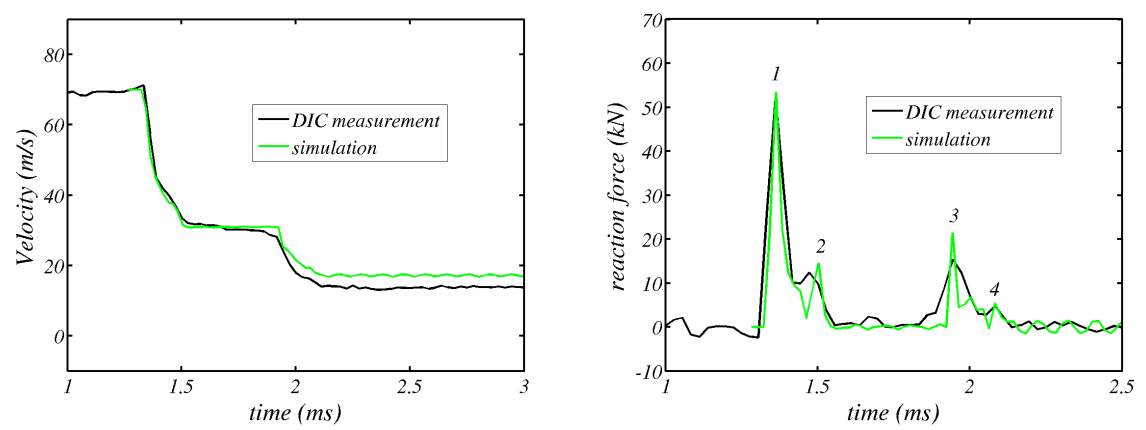

Fig. 11 Comparison of the simulated and measured $x$ component of the velocity (left) and impact force (right) corresponding to a frontal impact on an aluminium bar. In green, the simulation and, in black, the measurement with the proposed DIC technique

very good. The same number of peaks can be observed and their positions and amplitudes also match correclty.

\subsubsection{Physical interpretations of the impact force variation}

In the previous sections, attempts were made to validate the measurement method with either conventional measurement methods or numerical simulation. But each time, it was hard to draw definitive conclusions since these references include approximations and/or uncertainties and none is really representative of the ground truth. Here, a quantitative physical interpretation of the observations is proposed, in order to validate the measurement method with mechanical considerations. The time lapses between the different impact force peaks is compared to the first vibration eigenfrequencies of the target. The four peaks (numbered 1 to 4 in Figure 11(right)) correspond to an elastic deformation of the bar according to its first eigenmodes. If the bar is modeled by a free-free Euler-Bernouilli elastic beam, the angular eigenfrequencies $\omega$ are [23]:

$$
\omega_{n}=\frac{\alpha_{n}}{L^{2}} \sqrt{\frac{E I_{g z}}{\rho S}}, \quad \alpha_{n}=\{0,22.37,61.67,120.9, \ldots\}
$$

where $E, I_{g z}, \rho, S$ and $L$ are respectively the Young Modulus, second moment of the cross sectional area, density, cross sectional area and length of the beam. The first two symmetric bending modes are presented in Figure 12.

As a first approximation, it is assumed that the ball does not move between two impacts, so the time between two peaks corresponds to half the period associated 


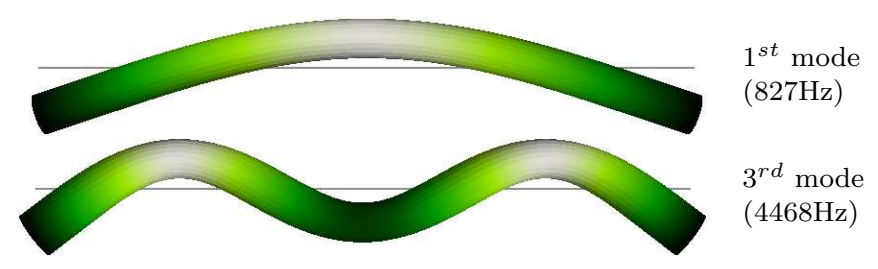

Fig. 12 First two symmetric mode shapes and corresponding eigenfrequencies of the bar.

with the eigenmodes triggered. Thus from the time period between peaks 1 and 3 $(0.594 \mathrm{~ms})$, it is possible to estimate the first eigenfrequency, $841 \mathrm{~Hz}$, as compared to the analytical value of $827 \mathrm{~Hz}$. More secondary rebounds (peaks 2 and 4 ) corresponding to the third eigenfrequency are observed. The period between peaks 1 and $2(0.124 \mathrm{~ms})$ provides an estimation of the third eigenfrequency of $4032 \mathrm{~Hz}$, whereas the analytical value is $4468 \mathrm{~Hz}$. Good agreement is observed between the analytical model and the estimation from the DIC-based tracking method. Not only is the uncertainty on the magnitude of the force reasonable, but the positions of the peaks also seem to provide reliable information.

Remark. The first eigenmode of the steel ball is higher than $80000 \mathrm{~Hz}$, which reinforces the hypothesis that the ball can be considered rigid, at the scale of the observations.

\section{Analysis of an oblique impact}

In this part, first results on oblique impacts are presented as an outlook for further studies. This kind of impact may appear on the lower surface of helicopter blades. The target was a steel plate $(200 \times 400 \times 1 \mathrm{~mm})$. The angle between the firing axis and the plate plane was $15^{\circ}$. The right and left edges of the specimen were simply supported, see Figure 13(left). For this test, the steel ball (19mm diameter and $28 \mathrm{~g}$ weight) was fired with an initial velocity of $80 \mathrm{~m} . \mathrm{s}^{-1}(\sim 90 \mathrm{~J})$. The impactor was covered by black and white speckles. The images were again aquired with a Photron digital camera. The frame rate, resolution and shutter speed were set to $20000 \mathrm{fps}, 512 \times 512 \mathrm{px}$ and $1 / 81000$ respectively.

The experimental DIC measurements are compared with a FE explicit simulation using the commercial software RADIOss. The impactor was modeled using a RADIOss spherical rigid wall. The plate was modeled using standard shell elements 

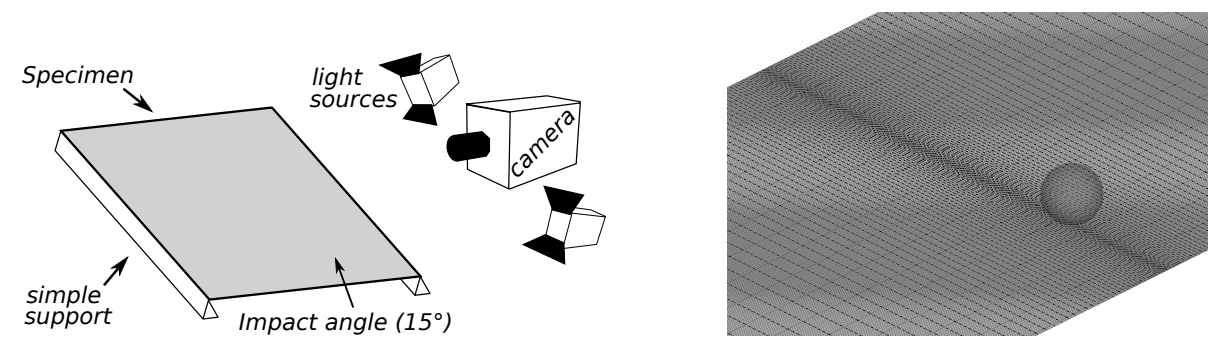

Fig. 13 (left) Setup of the oblique impact test on an inclined steel plate. Camera is on the side. (right) Corresponding modeling of the oblique impact test.

with hourglass stabilization. The mesh size was smaller in the impacted area (Figure 13(right)). The edge length varies from $0.5 \mathrm{~mm}$ to $10 \mathrm{~mm}$. The Johnson-Cook model was chosen for the material behaviour of the steel plate (see eq. (11)). The material characteristics are given in Table 5.

Table 5 Material parameters for the Johnson-Cook model chosen to model the steel plate.

\begin{tabular}{|c|c|c|c|c|c|c|c|c|c|}
\hline$E(G P a)$ & $\nu$ & $\dot{\varepsilon_{0}}$ & $a(M P a)$ & $b(M P a)$ & $c$ & $n$ & $m$ & $T_{i}(K)$ & $T_{\text {melt }}(K)$ \\
190 & 0.25 & 0.2 & 400 & 750 & 0.12 & 0.7 & 1 & 293 & 1700 \\
\hline
\end{tabular}

The velocities and impact forces are plotted in Figure 14. The variations of the velocity are smooth. By differentiation, the measured impact forces are a bit more noisy. The uncertainty on the acceleration is around $10 \%$ of the impact force magnitude. This test is not really representative of the oblique impact on helicopter blades, as the ball slides on the plate. Indeed almost no dissipation (thus no deceleration) was observed. Higher dissipations are expected with composite targets. However, a good agreement between the measured and simulated variations of impact forces and velocities is observed.

The main advantage of using DIC for this kind of impacts is that a measure can be performed even though the path of the projectile is not rectilinear (see Figure 15). Note that in this case, the deformation of the target results in projectile-totarget occlusions, with respect to which the proposed method seems robust. 

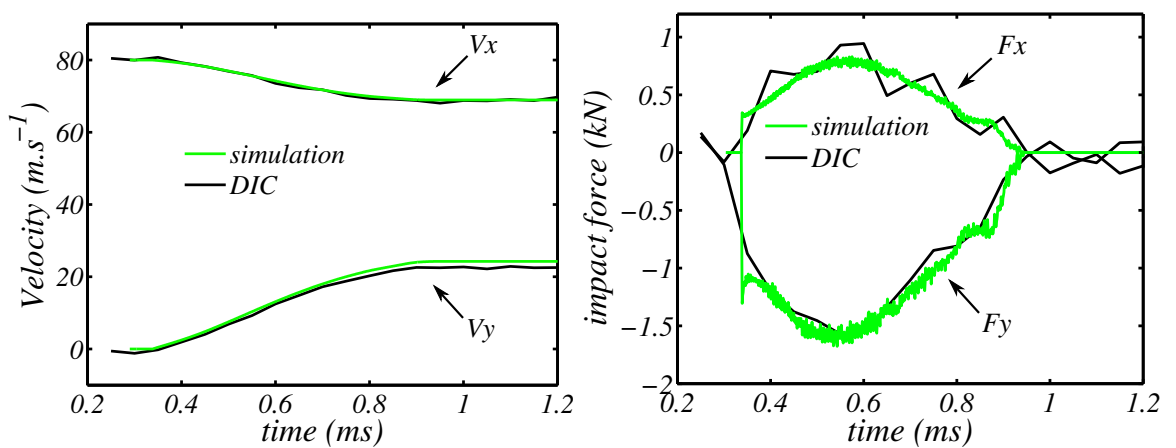

Fig. 14 Comparison of the simulated and measured $x$ and $y$ components of the velocity (left) and impact force (right) during an oblique impact on a steel plate. In green, the simulation and in black the measurement with the proposed DIC method

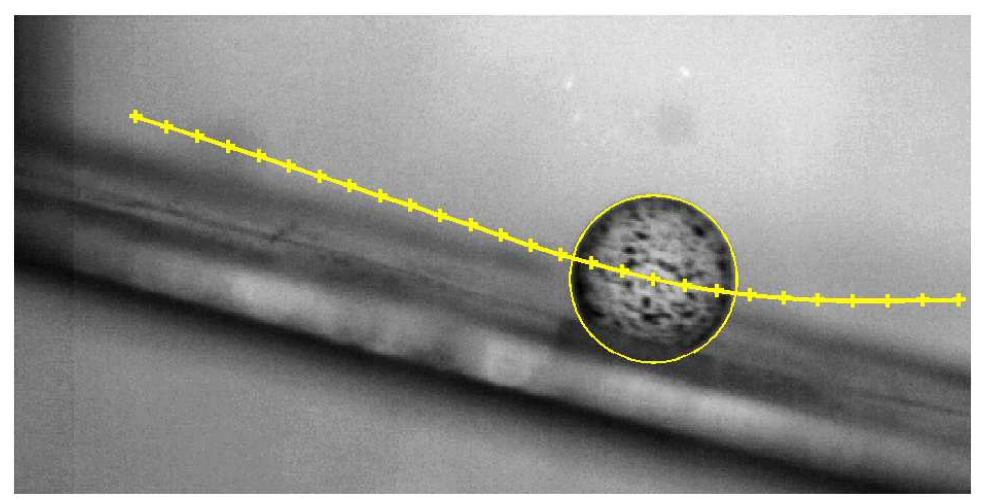

Fig. 15 Measured positions $(+)$ and position of the ball (circle) at $t=0.65 \mathrm{~ms}$ during the contact with the specimen.

\section{Conclusion}

A 2D Digital Image Correlation method was used to analyse the image sequence of medium velocity impacts of projectiles fired from gas-guns. The global interpolation of the displacement was based on five rigid body modes of the impactor in a circular region of interest, which made it possible to account for small rotations. It was shown that the method provided reliable estimations of the variation of velocities and impact forces during the impact. The method was also able to provide angular position and rotational velocities, which is of particular interest in many impact tests. Finally, this technique still works with non-rectilinear motions, which may appear in oblique impacts for instance. 
The method developed presents the following main advantages. Firstly, the technique presented does not need the projectile or the target to be instrumented. Secondly, the users of gas-gun devices usually already use high speed cameras to observe the behavior of the impacted targets qualitatively. Thus, the use of DIC techniques does not involve the purchase of other expensive measurement devices. Thirdly, this method allows the estimation of in-plane velocities and impact force even if the trajectory is curvilinear. Finally, the method is able to provide the three components of the rotation and angular velocity of the impactor, which could be useful for some quantitative analysis of impact experiments.

A priori uncertainty analyses were performed. It was shown that a more standard DIC technique based on translations only may over- or under-estimate displacement and velocity in case of rotation, which is detrimental to measurement accuracy. Thus the proposed interpolation that includes rotations constitutes a good alternative for the measurement of position, velocities and reaction forces as it is subject to much less bias. It has also been shown that it induce much less bias than a contour tracking algorithm such as the circular Hough transform.

The method was also used to analyse real experimental tests. In each case, the measurements were accurate. The estimated uncertainties were acceptable in both cases. Finally, the comparison with other image analysis techniques (Hough transform (new), Lucas-Kanade), other conventional measurement methods (light-gate and accelerometer) and reference numerical simulations revealed good measurement accuracies. This technique is thus a good alternative instrumentation for gas-gun experiments to measure translational and rotational velocity, acceleration, and impact force in the medium velocity range.

\section{References}

1. Abdulhamid, H., Kolopp, A., Bouvet, C., Rivallant, S.: Experimental and numerical study of AA5086-H111 aluminum plates subjected to impact. International Journal of Impact Engineering 51, 1-12 (2013)

2. Abrate, S.: Impact on composite structures. Cambridge University Press (1998)

3. Akhavan, F., Watkins, S.E., Chandrashekhara, K.: Prediction of impact contact forces of composite plates using fiber optic sensors and neural networks. Mechanics of Composite Materials and Structures 7(2), 195-205 (2000) 
4. Barker, L.M., Hollenbach, R.E.: Laser interferometer for measuring high velocities of any reflecting surface. J. Appl. Phys. 43, 4669-4675 (1972)

5. Besnard, G., Hild, F., Roux, S.: finite-element displacement fields analysis from digital images: Application to portevinle châtelier bands. Experimental Mechanics 46(6), 789803 (2006)

6. Bornert, M., Brémand, F., Doumalin, P., Dupré, J.C., Fazzini, M., Grédiac, M., Hild, F., Mistou, S., Molimard, J., Orteu, J.J., Robert, L., Surrel, Y., Vacher, P., Wattrisse, B.: Assessment of digital image correlation measurement errors: methodology and results. Experimental Mechanics 49(3), 353-370 (2009)

7. Castanié, B., Bouvet, C., Aminanda, Y., Barrau, J.J., Thevenet, P.: Modelling of low energy/low velocity impact on nomex honeycomb sandwich structures with metallic skins. International Journal of Impact Engineering 35, 620-634 (2008)

8. Chambon, S., Crouzil, A.: Dense matching using correlation : new measures that are robust near occlusions. In: Proceedings of British Machine Vision Conference (BMVC2003), vol. 42 , pp. 143-152 (2003)

9. Chang, C., Sun, C.: Determining transverse impact force on a composite laminate by signal deconvolution. Experimental Mechanics 29(4), 414-419 (1989)

10. Cheng, P., Sutton, M., Schreier, H., McNeill, S.R.: Full-field speckle pattern image correlation with b-spline deformation function. Experimental mechanics 42(3), 344-352 (2002)

11. Doyle, J.: Determining the contact force during the transverse impact of plates. Experimental Mechanics 27(1), 68-72 (1987)

12. Fedele, R., Galantucci, L., Ciani, A.: Global 2d digital image correlation for motion estimation in a finite element framework: a variational formulation and a regularized, pyramidal, multi-grid implementation. International Journal for Numerical Methods in Engineering 96(12), 739-762 (2013)

13. Fehrenbach, J., Masmoudi, M.: A fast algorithm for image registration. Comptes Rendus Mathématique 346(9-10), 593-598 (2008)

14. Fleming, D.C.: Delamination modeling of composites for improved crash analysis (1999)

15. Fuh, C., Maragos, P.: Motion displacement estimation using an affine model for matching. Optical Engineering 30(7) (1991)

16. Guinard, S., Allix, O., Guedra-Degeorges, D., Vinet, A.: A 3d damage analysis of lowvelocity impacts on laminated composites. Composites Science \& Technology 62(02), 585-589 (2002)

17. Hild, F., Roux, S.: Comparison of local and global approaches to digital image correlation. Experimental Mechanics 52(9), 1503-1519 (2012)

18. Horn, B., Schunck, G.: Determining optical flow. Artificial Intelligence 17, 185-203 (1981)

19. Hough, P.: Machine analysis of bubble chamber pictures. In: Int. Conf. High Energy Accelerators and Instrumentation (1959)

20. Hu, N., Fukunaga, H., Matsumoto, S., Yan, B., Peng, X.: An efficient approach for identifying impact force using embedded piezoelectric sensors. International Journal of Impact Engineering 34(7), 1258 - 1271 (2007) 
21. Inoue, H., Harrigan, J.J., Reid, S.R.: Review of inverse analysis for indirect measurement of impact force. Applied Mechanics Reviews 54(6), 503-524 (2001)

22. Johnson, A., Pickett, A., Rozycki, P.: Computational methods for predicting impact damage in composite structures. Composites Science and Technology 61(15), 2183-2192 (2001). DOI 10.1016/S0266-3538(01)00111-7

23. Lalanne, M., Berthier, P., Hagopian, J.D.: Mechanical Vibrations for Engineers. John Wiley \& Sons Inc (1983)

24. Leclerc, H., Périé, J.N., Roux, S., Hild, F.: Integrated digital image correlation for the identification of mechanical properties. Lectures Notes in Computer Sciences 5496, 161171 (2009)

25. Lin, H.Y., Gu, K.D., Chang, C.H.: Photo-consistent synthesis of motion blur and depthof-field effects with a real camera model. Image and Vision Computing 30(9), 605-618 (2012)

26. Lucas, B., Kanade, T.: An iterative image registration technique with an application to stereo vision. In: Proceedings of Imaging Understanding Workshop, pp. 121-130 (1981)

27. Navarro, P., Aubry, J., Marguet, S., Ferrero, J.F., Lemaire, S., Rauch, P.: Semi-continuous approach for the modelling of thin woven composite panels applied to oblique impacts on helicopter blades. Composites Part A: Applied Science and Manufacturing 43(6), 871-879 (2012)

28. Orteu, J.J., Garcia, D., Robert, L., Bugarin, F.: A speckle-texture image generator. In: Speckle'06 International Conference, vol. 6341, p. http://dx.doi.org/10.1117/12.695280 (2006)

29. Pan, B., Qian, K., Xie, H., Asundi, A.: Two-dimensional digital image correlation for in-plane displacement and strain measurement: a review. Measurement Science and Technology 20(6), 062,001 (2009)

30. Pan, B., Xie, H., Wang, Z.: Equivalence of digital image correlation criteria for pattern matching. Appl. Opt. 49(28), 5501-5509 (2010)

31. Pan, B., Yu, L., Wu, D.: High-accuracy 2d digital image correlation measurements with bilateral telecentric lenses: Error analysis and experimental verification. Experimental Mechanics 53(9), 1719-1733 (2013)

32. Passieux, J.C., Bugarin, F., David, C., Périé, J.N., Robert, L.: Multiscale displacement field measurement using digital image correlation: Application to the identification of elastic properties. Experimental Mechanics p. In press (2014). DOI 10.1007/s11340-014-9872-4

33. Passieux, J.C., Périé, J.N.: High resolution digital image correlation using proper generalized decomposition: PGD-DIC. International Journal for Numerical Methods in Engineering 92(6), 531-550 (2012)

34. Gomes Perini, L.A., Passieux, J.C., Périé, J.N.: A multigrid PGD-based algorithm for volumetric displacement fields measurements. Strain 50(4), 355 - 367 (2014) DOI 10.1111/str.12099

35. Réthoré, J., Morestin, F., Lafarge, L., Valverde, P.: 3D displacement measurements using a single camera. Optics and Lasers in Engineering 57(0), 20 - 27 (2014) 
36. Réthoré, J., Muhibullah, Elguedj, T., Coret, M., Chaudet, P., Combescure, A.: Robust identification of elasto-plastic constitutive law parameters from digital image using $3 \mathrm{~d}$ kinematics. Int. J. Solids and Structures 50, 73-85 (2013)

37. Reu, P., Miller, T.: The application of high-speed digital image correlation. The Journal of Strain Analysis for Engineering Design 43(8), 673-688 (2008)

38. Roscher, K.U., Fischer, W.J., Heinig, A., Pfeifer, G., Starke, E.: Start-up behavior of eventdriven sensor networks for impact load monitoring. Sensors and Actuators A: Physical 156(1), $109-114$ (2009)

39. Roux, S., Hild, F.: Stress intensity factor measurements from digital image correlation: post-processing and integrated approaches. Int J. Fract 140, 141-157 (2006)

40. Roux, S., Hild, F., Berthaud, Y.: Correlation image velocimetry : a spectral approach. Applied Optics 41(108-115) (2002)

41. Schreier, H., Braasch, J., Sutton, M.: Systematic errors in digital image correlation caused by intensity interpolation. Optical Engineering 39(11), 2915-2921 (2000)

42. Sun, Y., Pang, J., Wong, C.K., Su, F.: Finite element formulation for a digital image correlation method. Applied Optics 44(34), 7357-7363 (2005)

43. Sutton, M., Orteu, J.J., Schreier, H.: Image correlation for shape, motion and deformation measurements: Basic Concepts, Theory and Applications. Springer, New York, NY (USA) (2009)

44. Sutton, M., Wolters, W., Peters, W., Ranson, W., McNeill, S.: Determination of displacements using an improved digital correlation method. Image and Vision Computing 1(3), 133-139 (1983)

45. Takeda, S., Minakuchi, S., Okabe, Y., Takeda, N.: Delamination monitoring of laminated composites subjected to low-velocity impact using small-diameter fbg sensors. Composites Part A: Applied Science and Manufacturing 36(7), 903 - 908 (2005)

46. Talreja, R.: Damage and fatigue in composites a personal account. Composites Science and Technology 68(13), 2585-2591 (2008). DOI 10.1016/j.compscitech.2008.04.042

47. Vogler, T., Trott, W., Reinhart, W., Alexander, C., Furnish, M., Knudson, M., Chhabildas, L.: Using the line-VISAR to study multi-dimensional and mesoscale impact phenomena. International Journal of Impact Engineering 35(12), 1844-1852 (2008)

48. Wu, E., Tsai, T., Yen, C.: Two methods for determining impact-force history on elastic plates. Experimental Mechanics 35(1), 11-18 (1995)

49. Xu, G., Zhang, Z.: Epipolar geometry in Stereo, Motion and Object Recognition. Kluwer Academic Publishers (1996)

50. Yilmaz, A., Javed, O., Shah, M.: Object tracking: A survey. ACM Comput. Surv. 38(4) (2006) 